\title{
Dos contemporáneos: Ricardo Palma y Miguel Grau, figuras ilustres de la peruanidad'
}

Por Teodoro Hampe Martínez

I Agradezco la generosa colaboración del señor Jorge Cunza Arana, dilecto amigo, en la composición de este trabajo. 
Doctor en Historia por la Universidad Complutense de Madrid. Miembro Nacional Principal del Instituto Panamericano de Geografía e Historia y del Instituto Latinoamericano de Historia del Derecho. 
La idea de esta comunicación es poner en vinculación a dos emblemáticos personajes que en verdad son casi contemporáneos, prácticamente coetáneos. Anduve por ello dudando sobre el título exacto que merecería mi ponencia, y en un momento dado pensé ponerle «Palma y Grau, dos contemporáneos ante la paz y la guerra»; título un poco fuerte, algo atrevido, pero que alude a que ellos nacieron casi en el mismo año y que vivieron una época de paz, de relativa bonanza para el Perú, en la época del boom guanero, y luego experimentaron también los horrores de la guerra. Es la infausta guerra del Pacífico, que arrebata tempranamente la vida a Grau en 1879, a los 45 años de edad, y que afecta indirectamente a don Ricardo Palma (1833-1919) y a todos los peruanos; más aún porque Palma asumirá después la ímproba tarea de reconstruir la Biblioteca Nacional, afectada y saqueada por las tropas chilenas. ${ }^{2}$

El hecho cierto es que no hay una consonancia absoluta en los términos de vida, porque la biografía de Grau discurre originariamente en la región de Piura y las travesías marítimas, que inicia a muy temprana edad. En cambio, Palma es básicamente un hombre apegado a la ciudad de Lima, un intelectual de vida más sedentaria, y experimenta toda la posguerra del Pacífico hasta fallecer en 1919, cuarenta años

2 Cf. HAMPE MARTÍNEZ, Teodoro. «Nuevo asedio al "bibliotecario mendigo": Ricardo Palma en la Biblioteca Nacional», en Aula Palma (Lima), vol. 10, 2011 , p. 201-221. 
después que Grau. Desde ese punto de vista, si bien son casi contemporáneos en su nacimiento, desarrollan una trayectoria que solo en parte es coincidente.

\section{Acerca de Grau: el homenaje palmista}

He oído con atención las ponencias de los colegas de la mesa y entiendo que mi propuesta temática guarda sintonía básicamente con dos exposiciones. Uno es el discurso de incorporación de mi buen amigo el Dr. Juan Carlos Adriazola, que se ha referido a la vinculación de Palma con la casa natal de Miguel Grau en Piura, hoy día Casa Museo Gran Almirante Grau, perteneciente a la Marina de Guerra. En mi estudio recién publicado ${ }^{3}$, me ocupo tangencialmente de otra casa que también es museo, llamado de manera oficial Museo Naval Casa Grau, que está ubicado en la calle de Lescano, como se conoce en la terminología clásica a la primera cuadra del jirón Huancavelica. Esta es la residencia alquilada que habitó el matrimonio Grau-Cabero, y que pertenecía a la familia Osma-Ramírez de Arellano. ${ }^{4}$

Allí vivió por varios años don Miguel con la señora Dolores Cabero, su esposa, y su numerosa prole de diez hijos. Era el tiempo en que él transitaba por las viejas calles del centro de Lima y se desplazaba, seguramente a pie, desde esa residencia a la Cámara de Diputados, en su local primigenio ubicado donde antes había sido la Universidad de San Marcos, y que más o

3 HAMPE MARTÍNEZ, Teodoro. Miguel Grau, protagonista político. Piura: Municipalidad Provincial de Piura \& Caramanduca Editores, 2013. 177 p.p.

4 Se conserva una carta original del contralmirante Miguel Grau a don Javier de Osma y Ramírez de Arellano (general del Ejército y luego ministro de Estado), firmada a bordo del Huáscar, en Arica, 16 de setiembre de 1879. Este valioso documento, que estaba en manos privadas, fue donado al Congreso de la República en ceremonia realizada el 21 de julio de 2010. 
menos coincide con la ubicación actual del Palacio Legislativo. Ese era pues el tránsito cotidiano del Gran Almirante cuando no estaba en el Callao o a bordo de un buque, y esencialmente del emblemático navío que él comandó: el monitor Huáscar. ${ }^{5}$

Dadas estas condiciones, me aventuraría a decir que Palma es una especie de tributario de Grau, en el sentido de que él se aúna a la gran cantidad de personas, de escritores, de plumas célebres, que han rendido homenaje al ilustre marino.

He traído conmigo un libro del cual hay varios formatos y diversas ediciones, que es una valiosa compilación de cartas y facsímiles, de textos selectos y testimonios de diferentes autores en homenaje a Grau. Se llama A la gloria del Gran Almirante del Perú, Miguel Grau, y se debe al esfuerzo del almirante Fernando Casaretto Alvarado, actual director del Museo Naval del Callao. Entre varios otros textos, está incluido aquí un poema, la única composición directamente concerniente a Grau, de la pluma de Palma. ${ }^{6}$

No me atrevo a considerarme un especialista en poética o literatura, pero creo que la cualidad artística, o mejor dicho épica, de estos versos, no es de la mejor. En todo caso, es el genuino y sincero retrato de Palma sobre Grau, poco después de caído en Angamos, por lo cual le recogeré para traer la voz directa de don Ricardo en torno al marino piurano. Se llama simplemente "Miguel Grau" y dice así:

5 Para aclarar debidamente el ambiente hogareño y la filiación genealógica del héroe-parlamentario, contamos con el trabajo de Fernando GRAU UMLAUFF, «Entorno familiar de Miguel Grau Seminario» (discurso de incorporación al Instituto de Estudios Histórico-Marítimos del Perú, Lima, 20 de febrero de 2014).

6 CASARETtO AlVARADO, Fernando, ed. A la gloria del Gran Almirante del Perú, Miguel Grau. Lima: Marina de Guerra del Perú, Dirección de Intereses Marítimos, 2001, p. 131. 
Sol de resplandor fecundo

que nuestras pupilas hiere

es Miguel Grau... nunca muere

el astro-rey para el mundo.

¿A qué de duelo profundo

llanto derramar sincero

si ya, con buril de acero,

grabó ese nombre la Fama,

y el mundo la gloria aclama

del héroe y el caballero?

Vive la vida inmortal

que conquistó su heroísmo;

no conquistó en el abismo

del olvido nombre tal.

Del tiempo el giro fatal

dará más irradiación

a tan espléndida acción,

y del héroe la memoria

honra será de la historia,

gala de la tradición. ${ }^{7}$

Este es el personal homenaje que rinde Palma al heroísmo, la valentía y la caballerosidad de Grau, enalteciendo la memoria del prócer y contemporáneo suyo. No estaba muy lejos de la proposición encomiástica hecha por su archirrival político y literario, Manuel González Prada, al escribir en 1885 un ensayo sobre el héroe de Angamos. Señalaba este autor en la parte conclusiva de dicho texto: "En el combate homérico de uno contra siete, pudo Grau rendirse al enemigo; pero comprendió que por voluntad nacional estaba condenado a morir, que sus compatriotas no le habrían perdonado el

7 La misma composición está reproducida, bajo el título de "Hoja de laurel”, en AROSEMENA GARLAND, Geraldo. El almirante Miguel Grau. $7^{\mathrm{a}}$ ed. Lima: Banco de Crédito del Perú, 1979, p. 296. 
mendigar la vida en la escala de los vencedores...". Así lo ha recordado con acierto el investigador norteamericano William E. Arrillaga, en su trabajo sobre las repercusiones literarias de la guerra del Pacífico. ${ }^{8}$

\section{La dimensión política: (des)encuentros de Palma y Grau}

Al preparar esta comunicación me he topado con la feliz circunstancia de que hay abundancia de autores e investigadores, algunos inclusive bastante cercanos a mí por razón de amistad, cuyos aportes me han servido para elaborar el presente trabajo. En aras del tiempo y del espacio, anticiparé sucintamente la bibliografía -que va con detalle en el registro correspondiente, más abajo- para que se tenga una idea de qué clase de fuentes y autores he manejado al emprender esta aproximación a dos grandes figuras de la peruanidad y de la historia nacional del siglo XIX.

Está por ejemplo el documentado trabajo de un colega que en las presentes jornadas se incorpora al Instituto Ricardo Palma, el profesor Carlos A. Pérez Garay, que trata de Palma y la ideología y política de su tiempo. Es su tesis de licenciatura en Historia por la Universidad Nacional Mayor de San Marcos, del año 2010, que se titula de modo genérico «Liberalismo criollo». ${ }^{9}$

8 ARRILLAGA, William E. La guerra del Pacífico y su impacto en la literatura. Master's Theses, Paper ${ }^{\circ}$ 181. San Jose, CA: San Jose State University, 1991, p. 51.

9 PÉREZ GARAY, Carlos Alberto. Liberalismo criollo. Ricardo Palma, ideología y política: 1848-1919. Tesis (Lic. en Historia). Lima: Universidad Nacional Mayor de San Marcos, Facultad de Ciencias Sociales, 2010. 288 p. Este trabajo consta de cinco largos capítulos y examina la actividad política de Palma básicamente en tres partes: (1) su rol militante o activo, hasta la ascensión al mando de Balta; (2) su vinculación directa con el poder, como secretario de la Presidencia y miembro del Parlamento; y (3) su situación pasiva o expectante, en los últimos 45 años de su vida. 
He encontrado asimismo un artículo muy interesante del profesor Sandro Chiri Jaime (2013) sobre las tradiciones palmistas referidas a sucesos y personajes de la guerra del Pacífico, que no son en realidad muchas: apenas tres, que en dicho trabajo se analizan. Ellas están dedicadas a honrar singularmente a Francisco Bolognesi (tradición homónima), a Leoncio Prado ("Un montonero") y a los valerosos soldados que ofrendaron su vida en el morro de Arica ("La cajetilla de cigarros").

Otro investigador contemporáneo y con una obra notable, que cada día va creciendo más en volumen e importancia, es el profesor Rubén Quiroz Ávila, quien se ha ocupado (2009) del teatro peruano y de la temática de la guerra del Pacífico en obras dramáticas producidas desde el año 1879 en adelante. La profesora Nanda Leonardini, especialista en historia del arte, aborda a su vez (también en 2009) las coordenadas de identidad, ideología e iconografía en la República, reparando en obras emblemáticas de nuestro arte de los siglos XIX y XX, dedicadas a los grandes héroes de aquella guerra.

He encontrado asimismo una tesis de maestría de buena factura preparada por William E. Arrillaga el año 1991 en San José State University, sobre la guerra del Pacífico y su impacto en las literaturas del Perú, Chile y Bolivia. Complementariamente utilizo un artículo de la revista Consensus de la Universidad Femenina del Sagrado Corazón (2013), firmado por la profesora Ana María Lebrún, sobre las casas-museo de Grau y Palma ubicadas en Piura y Lima, respectivamente. Y entre varias otras contribuciones que podría citar está la de mi colega historiador de la Pontificia Universidad Católica del Perú, Carlos Contreras, que aporta una reflexión sobre memoria y verdad en las relaciones históricas entre Chile y Perú. ${ }^{10}$

10 Contreras se refiere al nuevo tono, de "acercamiento y romance", que han cobrado las relaciones peruano-chilenas en torno a la reciente coyuntura del 
Dentro de esta galería de autores no quiero dejar de mencionar al insigne Presidente fundador de nuestro Instituto, el Dr. Estuardo Núñez, quien el año 1945 en una tribuna académica de gran prestigio, la revista del Instituto Internacional de Literatura Iberoamericana, dedicó un artículo a don Ricardo Palma al cumplirse el primer cuarto de siglo de su muerte. Es interesante recoger de allí un extracto, porque son líneas en que el maestro Núñez vincula directamente a Palma con Miguel Grau:

Al cumplirse el vigésimo quinto aniversario de la desaparición de don Ricardo Palma, es deber de los peruanos -y acaso también de los americanos- meditar serenamente acerca de las virtualidades de la obra magnifica legada por ese esclarecido creador de arte. Han transcurrido ya los años en que su obra significativa, las Tradiciones peruanas, pudo juzgarse con más o menos prejuicio, con más o menos apasionamiento banderizante. [...] Palma tiene ya el rango de monumento nacional. Negarlo significaría lo mismo que negar la gloria de Miguel Grau o la estela heroica de Simón Bolívar, porque los valores de la acción, que es también espiritu, se confunden con los valores de la creación, cuando esta se vincula como la de Palma con el ser mismo de la raza y de la vida nacional. ${ }^{11}$

juicio sobre los límites marítimos, después de haberse mantenido ásperas y tirantes por más de 135 años. Cada vez que, con diversos propósitos, se había querido mejorar esas relaciones, se señalaba la imperiosa necesidad de corregir la memoria colectiva de la guerra de 1879. En consonancia con esta arquitectura de la memoria nacional -advierte el autor- se han levantado en la narrativa de la guerra algunos episodios de resonancia moral muy poderosa, de donde ha resultado que don Miguel Grau y don Ricardo Palma sean "las figuras predilectas de nuestro discurso histórico”. Cf. CONTRERAS, Carlos. «Memoria y verdad sobre el pasado chileno-peruano: una reflexión acerca de las posibilidades de "cambiar la historia"», en Coyuntura: análisis económico y social de actualidad (Lima), $\mathrm{n}^{\circ}$ 8, jul-ago. 2006, p. 11 .

11 NÚÑEZ, Estuardo. «A los 25 años de la desaparición de dos grandes escritores peruanos», en Revista Iberoamericana (Pittsburgh), vol. 9, nº 18, 1945, p. 287. 
Son ciertamente imperdibles esas frases, sesudas y contundentes, que ya desde aquel tiempo manifiestan la admiración de don Estuardo Núñez por nuestro mentor, el patriarca de las letras peruanas, patrono de la Universidad que lleva su nombre y de nuestro Instituto Ricardo Palma, que sigue sus huellas con devoción.

La otra vinculación que puedo señalar en apretada síntesis de lo que he oído esta noche, se refiere al trabajo presentado por el Dr. Roberto Reyes Tarazona acerca de la actividad política de Palma. Yo he desarrollado un esfuerzo parecido al ubicar a Miguel Grau, no en las coordenadas de marino, estratega o héroe de guerra, sino más bien de político militante. Esta es una dimensión que en verdad ya se sabía, en la cual varios autores habían reparado anteriormente, pues era conocido que había llevado la representación de la provincia de Paita en la Cámara de Diputados y había muerto incluso ejerciendo virtualmente esa función parlamentaria. ${ }^{12}$ Pero a nadie se le había ocurrido, o mejor dicho nadie había tenido las ganas que yo he tenido de zambullirme en los archivos del Congreso de la República y explorar toda la documentación que allí se guarda referente a sus intervenciones en el hemiciclo, sus proyectos de ley, sus propuestas generales, su vinculación con políticos importantes de la época y todo lo que constituye el trasfondo político de este personaje. ${ }^{13}$ Así crece la dimensión humana del Gran Almirante casi como en la célebre frase de Choquehuanca: "Con los

12 Cf. ORTIZ SOTELO, Jorge. Miguel Grau, el hombre y el mar. Lima: Fondo Editorial del Congreso del Perú, 2003, cap. 9 («Grau, el Huáscar y el Congreso»), especialmente la p. 165 y ss. El nombre del personaje figura en las listas de parlamentarios de esta obra: El Poder Legislativo y sus representantes, 1822-2000. Lima: Congreso de la República, 1998, p. 384 y 392.

13 Hace varios años, había adelantado un panorama de dicha investigación en el artículo titulado «El almirante Miguel Grau, la Marina de Guerra y la política peruana de la bonanza del guano (1854-1879)», en Anuario del Archivo y Biblioteca Nacionales de Bolivia (Sucre), vol. 7, 2001, p. 197-218. 
siglos crecerá vuestra gloria como crece la sombra cuando el sol declina"; y vemos en Grau al hombre múltiple, realmente ejemplar.

Respecto a la actividad política de ambos personajes, Palma y Grau, encontramos un punto en común, más allá del recinto parlamentario. No llegaron a coincidir en el Congreso Nacional porque cuando Palma ya estaba retirado de la política activa, luego de haber sido senador por Loreto por tres legislaturas sucesivas, se iniciará el encargo parlamentario de Grau. Pero sí estuvieron vinculados en un asunto sonado y bastante complejo, que ha llamado la atención de Oswaldo Holguín Callo, Carlos A. Pérez Garay y algunos otros estudiosos, referido a su participación en la rebelión anticastillista de 1856 . $^{14}$

Sucede que, como ustedes saben, ese año se había promulgado por insistencia de la facción política liberal una nueva Constitución del Estado, la cual no fue bien recibida en los ambientes conservadores y generó una reacción armada a través de un levantamiento que tuvo su instancia de origen (cómo no) en la ciudad de Arequipa. Ese movimiento recibió el apoyo de algunos buques de la Armada Peruana, como el Ucayali y el Loa, a bordo del cual estaba Ricardo Palma. En tal aventura, este buque se unirá luego con el Apurímac, que se hallaba a cargo del teniente segundo Lizardo Montero y tenía entre su tripulación al alférez de fragata Miguel Grau. Entonces Grau, Montero, Palma y otros personajes a quienes algunos ven como preclaros liberales van a compartir aquella intentona conservadora, de la

14 Véase el circunstanciado relato e interpretación de los hechos que ofrece HOlguín CAllo, Oswaldo. Tiempos de infancia y bohemia: Ricardo Palma (1833-1860). Lima: Pontificia Universidad Católica del Perú, Fondo Editorial, 1994, p. 516-532 y 596-601. Sostiene este académico que Palma "no estuvo acertado cuando en mala hora se plegó a los rebeldes", y que tal vez lo hizo presionado por las circunstancias o como mero imitador de la actitud de sus compañeros a bordo del vapor Loa. 
cual el profesor Pérez Garay no entiende bien el porqué. ${ }^{15}$ Y el presunto golpe termina desde luego en fracaso, pero llegando a vincular políticamente a nuestros protagonistas.

Tengo para mí, en una explicación un poco simplista, que no hay que ver las cosas de una manera radical o maniquea. Tanto Palma como Grau eran en esa época jóvenes que no habían llegado a los 25 años. ¿Quién no tiene derecho a cambiar de opiniones en su vida? ¿Qué grandes personajes de la actualidad o de tiempos antiguos no han variado sus conceptos políticos, su ideología, su percepción del mundo? De manera que no tiene nada de extraño que en aquel momento ellos hubieran abrazado la causa antiliberal; aunque en general se puede decir de Palma que, por sus escritos punzantes y su actitud crítica hacia el establishment social, favoreciera más bien la tendencia progresista.

No se puede afirmar lo mismo de Grau, en cambio. No llegaré a decir que era un gran conservador, pero sí tuvo (al revés de Palma) una intensa afiliación política, siendo prácticamente uno de los impulsores del Partido Civil. Fue muy amigo -y lejano pariente político- de don Manuel Pardo y Lavalle, el primer presidente civil del Perú; ambos eran rigurosamente contemporáneos, por haber nacido el mismo año de 1834, uno en Piura y otro en Lima. Tan vinculado estaba don Miguel con el político civilista que incluso llamará a Pardo cuando él se hallaba en Chile para que regrese al Perú a asumir la cabeza de su partido. ${ }^{16}$

15 Critica este autor la "actitud inconsecuente y acomodaticia" de José Toribio Pacheco, el poeta Toribio Mansilla y el propio Palma, que siendo liberales respaldaron la revolución del general Vivanco, caudillo militar de línea conservadora. Cf. PÉREZ GARAY, Liberalismo criollo, cit. [10], p. 76.

16 Es cierto que Grau no figura en la lista de fundadores de la Sociedad Independencia Electoral (1871), antecedente inmediato del Partido Civil; pero su labor en el núcleo dirigente de esta agrupación se hace cada vez más notoria después del gobierno de don Manuel Pardo. Véase al respecto MÜCKE, Ulrich. Der «Partido Civil» in Peru, 1871-1879. Zur Geschichte politischer Parteien und Repräsentation in Lateinamerika. Stuttgart: Franz Steiner Verlag, 1998, p. 137 y 337. 
En la legislatura ordinaria de 1878, Grau recibió en la Cámara de Diputados la doble responsabilidad de presidente de la comisión de Marina y miembro de la comisión auxiliar de Poderes, y fue uno de los oradores principales en el agasajo que se brindó al jefe del Partido Civil con motivo de su regreso a Lima. Sin embargo, a los pocos meses este caerá asesinado por un militar al salir del local del Senado, hoy Museo de la Inquisición y del Congreso. ${ }^{17}$

Este hecho luctuoso abre una gran incógnita, un vacío notable respecto al proyecto civilista que apuntaba hacia la reelección presidencial de Pardo en unas eventuales elecciones del año 1880, las cuales no se realizaron, obviamente, debido al curso de la contienda con Chile. ${ }^{18}$ En este punto hay que obrar muy finamente porque estamos ya en una perspectiva virtual, en otras palabras, contrafáctica. ¿Qué habría ocurrido si aquella conjunción de factores no se hubiera dado? Pero no quiero volar demasiado con la imaginación, sino comentar a ustedes que existe documentación que sustenta que Miguel Grau fue una especie de precandidato, producida la muerte de Pardo,

17 Comp. THORNDIKE, Guillermo. Grau, vol. 5. 1878: crimen perfecto. Lima: Fondo Editorial del Congreso del Perú \& Banco de Crédito del Perú, 2008, p. 470-482, donde se exponen las circunstancias del asesinato (16 de noviembre de 1878), y PANIAGUA, Valentín. Manuel Pardo y el Partido Civil. Apogeo y crisis del primer partido político en el Perú. Lima: Fondo Editorial del Congreso del Perú, 2009, p. 132-134, donde se analizan sucintamente los efectos de ese hecho para el movimiento civilista.

18 El asesinato de Pardo afectó profundamente a su amigo y camarada político, Grau, quien fue uno de los que cargaron el ataúd en las exequias y pronunció luego un sentido discurso necrológico, declarando las razones por las cuales la patria se hallaba en peligro. En la sesión congresal de 17 de noviembre de 1878, al día siguiente de aquel magnicidio, estuvo Grau entre los que votaron por la supresión de las garantías individuales. El presidente Mariano Ignacio Prado comprendió la gravedad del momento y llamó a un grupo selecto de parlamentarios del Partido Nacional y del Partido Civil, entre los cuales se encontraban Grau, Camilo N. Carrillo, Lizardo Montero y otros, para proponerles un pacto que evitara sumir al país en la anarquía. Cf. HAMPE MARTÍNEZ, Miguel Grau, protagonista político, cit. [3], p. 69. 
para sucederle ya sea como jefe del Partido Civil o como postulante a la máxima investidura en aquellas elecciones que presuntamente se hubieran dado a inicios de los años ochenta. ${ }^{19}$

La doctora Ella Dunbar Temple, insigne estudiosa de Grau y catedrática sanmarquina, encontró unas cartas que intercambia el Gran Almirante con su esposa, donde entre otras cosas le manifiesta a doña Dolores lo siguiente: "Había resuelto no contarte nada respecto al asunto Presidencia, porque francamente me parecía una broma, pero al ver que lo repites nuevamente con cierta seriedad, debo decirte que no pienso en tal cosa, por lo menos ahora que aún conservo mi razón..." (Carta del 27 de septiembre de 1879). ${ }^{20}$ Todo esto resulta muy interesante, aunque se mantiene en la esfera probabilista.

Pero lo que sí es verdad y se halla claramente abordado en mi libro reciente, es que Grau fue más allá del presunto lanzamiento a la jefatura del Estado e intentó asumir la presidencia de la Cámara de Diputados. El 27 de julio de 1879, estando a punto de embarcarse en sus últimas aventuras marítimas por aguas del Pacífico Sur, el comandante del Huáscar presenta su candidatura a la representación nacional, hecho que demuestra su clara vocación política. Sin embargo, pierde esa elección en segunda vuelta ante su compañero de

19 Se sabe que el 8 de febrero de 1879, tras un comunicado interno del Partido Civil, José de la Riva Agüero promovió una reunión de los senadores y diputados de esta agrupación, que contó con la asistencia de 80 personas, incluyendo al comandante Miguel Grau. Se trató entonces la cuestión del venidero proceso electoral de 1880 y se barajaron nombres para una eventual candidatura presidencial. Véase la documentación que recoge PUENTE CANDAMO, José Agustín de la. Miguel Grau. Lima: Instituto de Estudios Histórico-Marítimos del Perú, 2003, p. 255-256, nota 9.

20 TEMPLE, Ella Dunbar. «El "victorial” de Miguel Grau». Separata de la revista San Marcos (Lima), vol. 20, 1979, p. 22. 
armas, el comandante Camilo N. Carrillo. Al enterarse de este fracaso, mientras estaba con su buque fondeado en el puerto de Arica, hace llegar a las autoridades del Congreso un oficio por el cual solicita ser relevado de sus tareas como diputado por Paita, a causa de las incumbencias de la guerra. ${ }^{21}$

¿Qué habría ocurrido si el héroe de Angamos hubiera sido electo presidente de la Cámara? No podemos saberlo con certeza: es una cuestión difícil de responder.
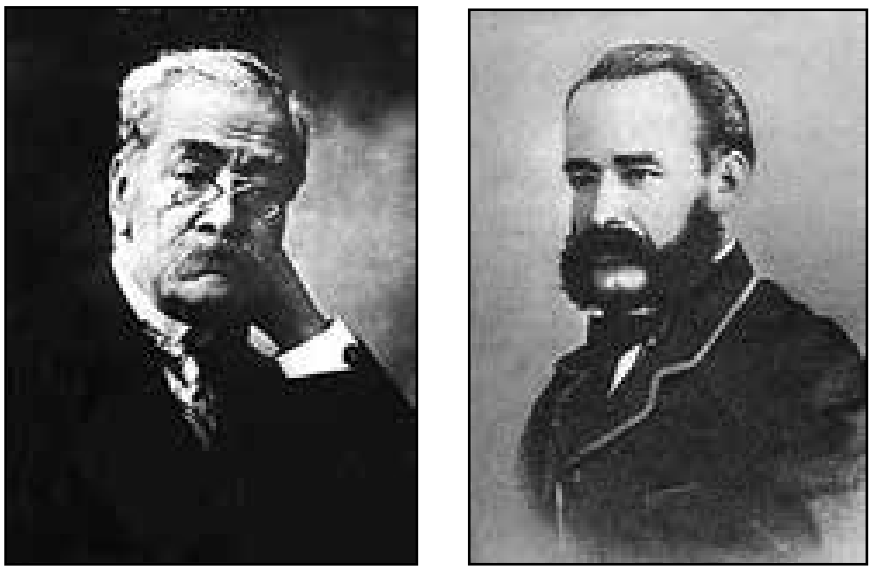

Dos contemporáneos: Ricardo Palma y Miguel Grau, figuras ilustres de la peruanidad.

21 Archivo General del Congreso de la República, Actas de la Cámara de Diputados, 35-B, vol. II, p. 110-1 I1. Refiere el documento textualmente: "En seguida [el Presidente] manifestó que, no habiendo obtenido el Sr. Carrillo las dos terceras partes de sufragios, requisito indispensable para ser reelecto en el cargo de Presidente (...), se iba a proceder a una nueva elección entre el expresado Sr. Carrillo y el Sr. Grau; y suspendió la sesión. Practicado el escrutinio, obtuvieron el Sr. Carrillo, 55 votos; el Sr. Grau, 20 votos; viciado, 1 voto". Para más detalle, véase HAMPE MARTÍNEZ, Miguel Grau, protagonista político, cit. [3], p. 70 y 127. 


\section{A manera de conclusión}

Y por último, trato de plantear aquí: ¿Qué se puede decir de nuestro laureado escritor, don Ricardo Palma, y su interpretación sobre la guerra del Pacífico a través de las obras que él publicó?

La brevísima producción textual de Palma sobre esa contienda lleva el sello indeleble del testigo de una época atroz, la huella del dolor de haber sido víctima de los funestos acontecimientos, junto con la patriótica intención de encomiar el esfuerzo y el valor de los que entregaron sus vidas por la defensa del Perú. No se descarta entonces el fuego de la pasión en las historias que emite un narrador que acota, aclara y arremete a favor de la causa peruana, según ha advertido lúcidamente el poeta y crítico literario Sandro Chiri Jaime. ${ }^{22}$

En tal sentido, para Palma recordar es negarse al olvido: se desata la memoria no por rencor sino para valorar a la nación como ente real y vivo. En el fondo, los breves relatos que aluden a la guerra del Pacífico (las tradiciones nombradas: "Francisco Bolognesi", "Un montonero" y "La cajetilla de cigarros") no trasuntan odio ni resentimiento, sino una llamada de atención a los peruanos de todos los tiempos para que actúen según el modelo de Grau, Bolognesi, Ugarte, Leoncio Prado y los demás héroes que ofrendaron sus vidas por la patria. Se trata de un discurso de entraña nacionalista que persigue unificar al país en un contexto -el período de la Reconstrucción Nacional- de desánimo generalizado.

Se ha dicho, en este contexto, que el nacionalismo y el patriotismo de González Prada en sus acerbas declaraciones

22 CHIRI JAIME, Sandro. «Tradiciones de la guerra del Pacífico (1879-1883)», en Nueva corónica (Lima), nº 2, jul. 2013, p. 440. 
sobre la guerra de 1879 quedaron reflejados en la obra de otros dos autores peruanos, Palma y José Santos Chocano. ${ }^{23}$ Todos ellos utilizaron ejemplos de esa conflagración para llamar al patriotismo a sus conciudadanos. Tanto el tradicionista como Chocano narraron episodios de la batalla de Arica, por ejemplo, para exaltar a los soldados que se sacrificaron en vez de rendirse a los invasores chilenos.

En fin, las tradiciones que arriba hemos mencionado evidencian un sentimiento que apuesta por la regeneración del país y se condice con la política de reconstrucción que impulsaron sucesivos gobiernos después de la guerra con Chile, especialmente los de Cáceres, Piérola y López de Romaña. En este caso, "la narrativa literaria acompaña el discurso oficial y el impulso renovador que se afana, como tarea inmediata, por rehacer el país desde los escombros que dejó la trágica contienda" (Chiri Jaime). ${ }^{24}$

Así se puede resumir la interpretación que brindan las tradiciones palmistas sobre la guerra del guano y el salitre. En ese discurso, el Perú exhibe héroes que no se rindieron, que lucharon hasta quemar el último cartucho, aun en inferioridad de condiciones bélicas. Aparecen hombres -y casi nada mujeresque supieron afrontar con honor e hidalguía la defensa de la patria, a costa de sus vidas y de la humillante derrota. Pero no se trata de muertes ni de afanes inútiles, parecen decir los relatos citados, pues el Perú se consolidará como nación después del tratado de Ancón. Este es el firme y esperanzador legado que don Ricardo Palma entrega a nosotros, sus lectores del siglo XXI.

23 ARRILLAGA, La guerra del Pacífico y su impacto en la literatura, cit. [9], p. 49-51.

24 CHIRI JAIME, «Tradiciones de la guerra del Pacífico (1879-1883)», cit. [23], p. 455 . 


\section{Bibliografía}

AROSEMENA GARLAND, Geraldo. "El almirante Miguel Grau". $7^{\mathrm{a}}$ ed. Lima: Banco de Crédito del Perú, 1979. xxxii, 306 p.

ARRILLAGA, William E. "La guerra del Pacífico y su impacto en la literatura". Master's Theses, Paper $n^{\circ}$ 181. San Jose, CA: San Jose State University, 1991. iv, 83 p.

CASARETTO ALVARADO, Fernando, ed. "A la gloria del Gran Almirante del Perú, Miguel Grau". Lima: Marina de Guerra del Perú, Dirección de Intereses Marítimos, 2001. 361 p.

CHIRI JAIME, Sandro. "Tradiciones de la guerra del Pacífico (1879-1883)", en Nueva corónica (Lima), nº 2, jul. 2013, p. 439-460.

CONGRESO DE LA REPÚBLICA. El Poder Legislativo y sus representantes, 1822-2000. Lima: Congreso de la República, 1998. viii, 917 p.

CONTRERAS, Carlos. «Memoria y verdad sobre el pasado chileno-peruano: una reflexión acerca de las posibilidades de "cambiar la historia" ", en Coyuntura: análisis económico y social de actualidad (Lima), $\mathrm{n}^{\mathrm{0}}$ 8, jul-ago. 2006, p. 10-11.

HAMPE MARTÍNEZ, Teodoro. "El almirante Miguel Grau, la Marina de Guerra y la política peruana de la bonanza del guano (1854-1879)", en Anuario del Archivo y Biblioteca Nacional de Bolivia (Sucre), vol. 7, 2001, p. 197-218.

HAMPE MARTÍNEZ, Teodoro. «Nuevo asedio al "bibliotecario mendigo": Ricardo Palma en la Biblioteca Nacional», en Aula Palma (Lima), vol. 10, 2011, p. 201-221.

HAMPE MARTÍNEZ, Teodoro. "Miguel Grau, protagonista político". Piura: Municipalidad Provincial de Piura \& Caramanduca Editores, 2013. 177 p.

HOLGUÍN CALLO, Oswaldo. Tiempos de infancia y bohemia: Ricardo Palma (1833-1860). Lima: Pontificia Universidad Católica del Perú, Fondo Editorial, 1994. 735 p. 
LEBRÚN ASPÍLLAGA, Ana María. "Las casas-museo como un puente hacia la comunidad en los tres períodos de la historia del Perú", en Consensus (Lima), vol. 18, n², 2013, p. 23-38.

LEONARDINI, Nanda. "Identidad, ideología e iconografía republicana en el Perú”, en Arbor: revista de ciencia, pensamiento y cultura (Madrid), vol. 185, n 740, 2009, p. 1259-1270.

MÜCKE, Ulrich. Der «Partido Civil» in Peru, 1871-1879. Zur Geschichte politischer Parteien und Repräsentation in Lateinamerika. Stuttgart: Franz Steiner Verlag, 1998. 384 p.

NÚÑ̃EZ, Estuardo. "A los 25 años de la desaparición de dos grandes escritores peruanos [Palma y Valdelomar]”, en Revista Iberoamericana (Pittsburgh), vol. 9, no 18, 1945, p. 287-296.

ORTIZ SOTELO, Jorge. "Miguel Grau, el hombre y el mar". Lima: Fondo Editorial del Congreso del Perú, 2003. 283 p.

PANIAGUA, Valentín. "Manuel Pardo y el Partido Civil. Apogeo y crisis del primer partido político en el Perú". Lima: Fondo Editorial del Congreso del Perú, 2009. 242 p.

PÉREZ GARAY, Carlos Alberto. "Liberalismo criollo. Ricardo Palma, ideología y política: 1848-1919”. Tesis (Lic. en Historia). Lima: Universidad Nacional Mayor de San Marcos, Facultad de Ciencias Sociales, 2010. 288 p.

PUENTE CANDAMO, José Agustín de la. "Miguel Grau”. Lima: Instituto de Estudios Histórico-Marítimos del Perú, 2003. xlviii, 573 p.

QUIROZ ÁVILA, Rubén. "Cuatro obras sobre la guerra del Pacífico en el teatro peruano", en Desde el Sur (Lima), vol. 1, n 2, Lima, may-oct. 2009, p. 315-335.

TEMPLE, Ella Dunbar. «El "victorial" de Miguel Grau». Separata de la revista San Marcos (Lima), vol. 20, 1979. 51 p.

THORNDIKE, Guillermo. "Grau", vol. 5. 1878: crimen perfecto. Lima: Fondo Editorial del Congreso del Perú \& Banco de Crédito del Perú, 2008. xvi, 524 p. 